\title{
Experimental Validation of a Robotic Stretcher for Casualty Evacuation in a Man-Made Disaster Exercise
}

\author{
Anthony Mandow, Javier Serón, Francisco Pastor, and Alfonso García-Cerezo ${ }^{1}$
}

\begin{abstract}
This paper describes a cooperative search and rescue exercise where an unmanned ground vehicle (UGV) is used by a military rescue team for extraction and evacuation of a casualty from an unsafe man-made disaster area. This experimental validation was performed within a full-scale emergency response exercise organized on June 2019 by the Chair of Safety, Emergencies and Disasters at Universidad de Málaga (Spain). With this purpose, we adapted the skidsteer Rambler robot to carry a stretcher with appropriate roll-in and locking mechanisms. The mission consisted of two phases: first, extraction from the hot zone was performed with remote teleoperation using a dummy; second, casualty evacuation (CASEVAC) to an aeromedical evacuation point was done with sightline teleoperation moving an actual volunteer. The realistic one-shot exercise was performed by actual rescue personnel with no previous experience with the robotic system. The paper shares insight and lessons learned from this concept validation experience.
\end{abstract}

Keywords - Disaster robotics; Search and rescue robots; Unmanned Ground Vehicles; Casualty evacuation.

\section{INTRODUCTION}

Using unmanned ground vehicles (UGV) for victim extraction from unsafe areas in natural and man-made disasters is a key application of rescue robotics [1]. The support of robotic systems for casualty extraction and casualty evacuation (CASEVAC) can increase the operational flexibility, act as force multiplier in high demand situations, and reduce risks for first responders in both civil and military scenarios.

However, not so many works have addressed casualty evacuation and extraction [2]. Furthermore, existing solutions pose interesting research challenges, as analyzed in a recent review by Williams et al. [3]. Some researchers have tackled manipulation aspects of casualty extraction, such as placing the hook to tow the victim to a safe area [2] or lifting the person with a dual arm [4]. However, manipulation of a casualty can cause additional damage, such as neck or spinal chord injuries. Thus, other works have focused on simpler and safer procedures based on stretchers.

Large robotic multipurpose equipment transport platforms developed for military applications have offered the possibility of carrying multiple stretchers for casualty evacuation [3]. On the other hand, the works of Iwano et al. [5] [6] aimed at helping first responders to extract and move casualties

\footnotetext{
* This work was partially supported by the project "TRUST-ROB: Towards Resilient UGV and UAV Manipulator Teams for Robotic Search and Rescue Tasks", funded by the Spanish Government (RTI2018-093421B-I00).

${ }^{1}$ Universidad de Málaga, Robotics and Mechatronics Group, Andalucía Tech, 29071 Málaga, Spain. amandow@uma.es, ajgarcia@uma.es
}

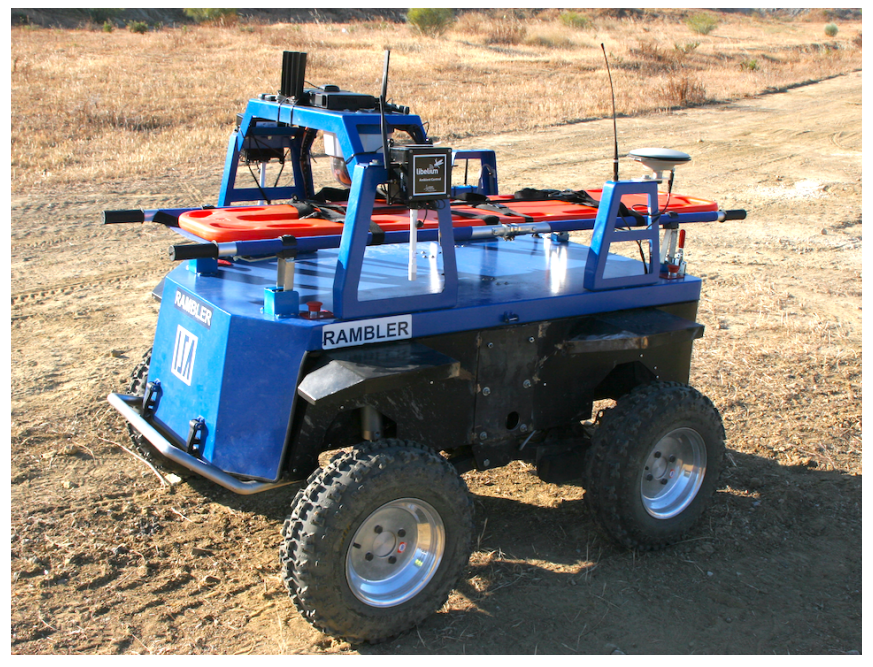

Fig. 1. All-terrain Rambler robot with the adapted detachable onboard stretcher.

with a stretcher-like robot platform with a conveyor system, a concept that has been extended in other works [7].

In this work, our major goal was to test a robotic concept that can be accepted and integrated in a straightforward way into the procedures of actual rescue personnel. Thus, we do not address casualty body manipulation. Instead, we adapted a highly maneuverable all-terrain UGV (see Fig. 1) so that rescuers could easily attach the stretcher for safe transportation of the casualty in an unstructured disaster environment. To the best of our knowledge, this is the first robotics research work that documents and discusses the use of a stretcher robotic system in a casualty extraction and evacuation scenario performed by actual first-rescuers in a realistic exercise without previous training.

The remaining of the paper is organized as follows. Section II provides context and the objective of the exercise. Section III describes the robotic system. Section IV discusses how the exercise was developed and offers some insight and lessons learned. Finally, Section $\mathrm{V}]$ is for the conclusions.

\section{Evacuation Mission Overview}

The UGV casualty extraction and evacuation scenario was part of a large-scale disaster response exercise conducted in Málaga (Spain) on June 6, 2019. The exercise involved a series of scenarios corresponding to a man-made disaster and was organized by the Chair of Safety, Emergencies and Disasters at Universidad de Málaga (UMA).

An aerial view of the exercise area with a layout of the robotic stretcher mission is shown in Fig. 2. The first 